\title{
LHCb long term plans beyond the current upgrade.
}

\author{
Frederic Teubert* ${ }^{* \dagger}$ \\ CERN, EP department \\ E-mail: frederic.teubertecern.ch
}

HL-LHC can provide up to $2 \times 10^{34} \mathrm{~Hz} \mathrm{~cm}^{-2}$ instantaneous luminosity to LHCb without significant loses for the other interaction points at the LHC ring, and be able to integrate up to $300 \mathrm{fb}^{-1}$ before the insertion magnets need replacement. There is a clear and strong physics case for an LHCb second upgrade (LHCb phase 2 upgrade) during the long shutdown currently scheduled in 2030. Very rare decays, or exotic searches are limited by statistics. Some of the most interesting CKM measurements are not limited by systematic uncertainties. Some of the improved detector technologies needed for this second upgrade, and other ideas discussed in these proceedings, could already be implemented during the long shutdown in 2024-2025 (LHCb phase 1b upgrade).

16th International Conference on B-Physics at Frontier Machines

2-6 May 2016

Marseille, France

* Speaker.

$\dagger$ On behalf of the LHCb Collaboration. 


\section{Introduction}

LHCb has been very successful in producing a wealth of physics results from the $3 \mathrm{fb}^{-1}$ accumulated at RUN-I (2010-2012) spanning from (very) rare decays of $\mathrm{B}, \mathrm{D}, \mathrm{K}$-mesons and $\tau$ leptons [1], to precise measurements of $\mathrm{CP}$ violating phases in the CKM couplings [2], to the discovery of new exotic hadrons [3] or even going beyond the original design purpose providing precise measurements of electroweak observables or searches for new particles in the forward direction. RUN-2 (2015-2018), with its increase in energy provides a proportional increase in heavy flavor production, effectively doubling the statistics available per unit of luminosity. LHCb expects to accumulate another $\sim 5 \mathrm{fb}^{-1}$ during RUN-2 running at an average luminosity $\langle\mathscr{L}\rangle$ $\sim 4 \times 10^{32} \mathrm{~Hz} \mathrm{~cm}^{-2}$ and average pileup $<\mu>\sim 1$. After RUN2, LHCb will upgrade the front-end electronics (and most of the sub-detectors) to allow for a full readout of the detector at a rate of 40 MHz. This upgrade [4] will allow for a more efficient trigger, in particular in hadronic final states, and an increase of the instantaneous average luminosity at LHCb by a factor $\sim 5$, to be able to accumulate a total of $\sim 50 \mathrm{fb}^{-1}$ during RUN-3 (2021-2023) and RUN-4 (2026-2029).

In this time scale Belle-II expects to start data taking by the end of 2018 and to accumulate $50 \mathrm{ab}^{-1}$ after six years. By then, during the Long Shutdown after RUN-3 (LS3), LHC will be replaced by HL-LHC to be able to increase the instantaneous average luminosity at ATLAS and CMS to $\left\langle\mathscr{L}>\sim 5 \times 10^{34} \mathrm{~Hz} \mathrm{~cm}^{-2}\right.$ and $\langle\mu>\sim 140$, while these two general purpose detectors will also be upgraded, not only to deal with the harsher HL-LHC environment, but in some aspects, to provide an even better performance as for instance b/c-tagging capabilities.

In this context it is natural to consider a second LHCb upgrade after RUN4, i.e. during LS4 (2030), when the first upgrade will have reached its target integrated luminosity and the HL-LHC will also be capable of providing a much higher instantaneous luminosity. The unique $\mathrm{LHCb}$ acceptance for low transverse momentum $\left(\mathrm{P}_{\mathrm{T}}\right)$ decay products provides an unmissable opportunity to maximize the physics output from HL-LHC, which happens to be the highest priority of the European High Energy Physics Community as described by the European Strategy Update [5].

LHCb is also considering moderate cost improvements to the upgraded detector during LS3 which have the potential to extend the physics capabilities denoted as a "phase 1b" upgrade. Examples of these potential improvements are:

- replace the Hadronic Calorimeter (HCAL) with a new iron shielding for the muon chambers, as well as replacing the innermost muon detectors with new technologies.

- replace the innermost Electromagnetic Calorimeter (ECAL) region with new technologies, as they need to be replaced anyway due to radiation damage.

- replace the innermost SciFi tracker region with a new tracker detector.

- use new technologies such as a TORCH [6] as a new hadron identification detector or as a fast timing device, perhaps embedded in the ECAL to help with pileup mitigation.

- increase tracking acceptance for low momentum particles by installing tracking stations along the dipole magnet internal side. 
In the rest of this paper I will focus on the physics case for the LHCb second upgrade during LS4, the studies made by the HL-LHC experts in terms of performance and protection of the interaction region and few preliminary studies on what kind of detector modifications would be required.

\section{The physics case}

LHCb is designed to reach much lower $\mathrm{P}_{\mathrm{T}}$ thresholds than ATLAS or CMS, which allows for a large acceptance of decay products of relatively light particles. The current LHCb detector, and the first upgrade during LS2, have been designed to deal with relatively low pileup $(\mu<5)$. If a second upgrade of the $\mathrm{LHCb}$ detector is able to absorb much higher pileup $\mathscr{O}(50)$, then an increase of the instantaneous luminosity by a factor $\sim 50$ w.r.t. RUN-2 is feasible (see section 3 ). Very rare decays of light mesons, $\tau$-leptons and searches for exotic new particles are limited by the statistics available and will certainly benefit of such a significant increase in integrated luminosity. Similarly, some of the most interesting measurements of the CKM phases are not limited by systematic uncertainties, and depending on the ability to perform time dependent analysis (see section 4) it could be possible to reach unprecedented precision in testing the CKM paradigm.

To illustrate the generic argument above, I would like to give a few explicit examples of potentially very interesting measurements:

- The very rare decay $\mathrm{B}_{\mathrm{s}} \rightarrow \mu^{+} \mu^{-}$has been successfully observed at the LHC, and its branching ratio measured [7] with a precision of $23 \%$ consistent with the SM predictions. The rate of the decay $\mathrm{B}_{\mathrm{d}} \rightarrow \mu^{+} \mu^{-}$is suppressed by a factor $\left|V_{t d} / V_{t s}\right|^{2}$ in the SM, and no significant signal has been established as of today. By the end of RUN-4, LHCb (CMS) with 50 (1000) $\mathrm{fb}^{-1}$ expect to have $\sim 70(150) \mathrm{B}_{\mathrm{d}}$ and $\sim 650$ (1400) $\mathrm{B}_{\mathrm{s}}$ dimuon candidates with $\mathrm{S} / \mathrm{B} \sim 1.5$ for $\mathrm{B}_{\mathrm{d}}$ and $\sim 3$ for $\mathrm{B}_{\mathrm{s}}$ decays for both experiments assuming the SM predictions, allowing for a $40(30) \%$ measurement of the ratio $\mathrm{R}=\mathscr{B}\left(\mathrm{B}_{\mathrm{d}} \rightarrow \mu^{+} \mu^{-}\right) / \mathscr{B}\left(\mathrm{B}_{\mathrm{s}} \rightarrow \mu^{+} \mu^{-}\right)$. This ratio $(\mathrm{R})$ is a very powerful tool to constrain the flavor structure of models beyond the SM. LHCb has a better invariant mass resolution and hadron particle identification capabilities which allows for a better control of systematic uncertainties. After the LHCb second upgrade with $300 \mathrm{fb}^{-1}$ integrated luminosity and in combination with the expected CMS sensitivity [8] with $3000 \mathrm{fb}^{-1}$, we could reach a $14 \%$ combined precision in this ratio. Moreover the larger number of $\mathrm{B}_{\mathrm{s}}$ dimuon decays will allow the study of new observables such as the effective lifetime or $\mathrm{CP}$ asymmetries.

- The LHC collider produces an enormous quantity of charm and beauty mesons and subsequently of $\tau$ leptons from their decays at a rate (in RUN-1) five orders of magnitude bigger than at the $\mathrm{e}^{+} \mathrm{e}^{-} \mathrm{B}$-factories. The luminosity accumulated at the B-factories was three orders of magnitude bigger than what LHCb accumulated in RUN-I, and the analysis efficiency and background levels at $\mathrm{LHCb}$ are worse compensating for the much larger production rate. Nonetheless, LHCb has produced limits [9] with RUN-I data $\left(\sim 10^{11} \tau\right)$ on the process $\tau^{ \pm} \rightarrow \mu^{+} \mu^{-} \mu^{ \pm}$very close to the ones obtained by BaBar and Belle $\left(\sim 10^{9} \tau\right)$ [10], resulting in a world average of $\mathscr{B}\left(\tau^{ \pm} \rightarrow \mu^{+} \mu^{-} \mu^{ \pm}\right)<1.2 \times 10^{-8}$ at $90 \%$ C.L. LHCb expects to improve on the B-factories results by the end of RUN-2. In many theories [11] beyond the SM it 
is quite possible to expect this branching ratio to be in the region $\left(10^{-9}-10^{-8}\right)$. Belle-II will be exactly covering this region of sensitivity when accumulating up to $50 \mathrm{ab}^{-1}$. If a signal is seen by Belle-II, a phase-2/second upgrade of LHCb with $300 \mathrm{fb}^{-1}$ integrated luminosity will be the only possibility to confirm with an independent experiment such a discovery, or if the search is negative to improve the limits by combining Belle-II and LHCb results.

- The SM does not predict the values of the CKM couplings, they all need to be measured experimentally. If we assume NP is mostly affecting measurements in loop-dominated processes, a precise measurement of the phase of $V_{u b}$ (to a very good approximation equivalent to $\gamma$ in the unitary triangle) is required to be used as SM reference value in comparisons with loop-dominated measurements. The uncertainty in $\gamma$ after RUN4 is expected to reach $\sim 0.9^{\circ}$ mainly using time-independent analysis of $\mathrm{B} \rightarrow \mathrm{DX}$ decays where loop contributions and theoretical uncertainties are negligible. With LHCb second upgrade $\left(300 \mathrm{fb}^{-1}\right)$ and using all available modes this uncertainty is reduced to $\sim 0.4^{\circ}$, which will provide a very stringent test of new phases contributing in $\mathrm{B}_{\mathrm{d}}$ decays.

- If time-dependent analyses are possible with a pileup $\mathscr{O}(50)$, then a precise measurement of the phase of $V_{t s}$ (to a very good approximation equivalent to $\phi_{s}$ in the unitary triangle) is possible at an LHCb second upgrade. The uncertainty on $\phi_{s}$ after RUN4 is expected to reach $9 \mathrm{mrad}$ using mainly the time-dependent analysis of the decay $\mathrm{B}_{\mathrm{s}} \rightarrow \mathrm{J} / \psi \phi$. If new phases are affecting differently $B_{s}$ and $B_{d}$ mixing it could very well be that a comparison of loop-dominated processes in $\mathrm{B}_{\mathrm{s}}$ and $\mathrm{B}_{\mathrm{d}}$ mixing with tree level measurements provides a different picture. With an LHCb second upgrade $\left(300 \mathrm{fb}^{-1}\right)$, and including other $\mathrm{LHCb}$ measurements, as well as potential ATLAS and CMS contributions, the uncertainty in $\phi_{s}$ is expected to be reduced to $\sim 2 \mathrm{mrad}$.

- Following the first LHCb upgrade in LS3, the detector will be fully readout at $40 \mathrm{MHz}$ allowing for a very flexible full software trigger. This, in addition to the excellent detector resolution, opens up new possibilities for searches, like the search for dark photons using the inclusive dimuon mass spectrum [12]. In Figure 1 one can see the LHCb potential to look for such dark photons in the plane of the mass of the dark photon vs the coupling strenght as described in reference [12]. The improvement from accumulating more luminosity (16, 50, $500 \mathrm{fb}^{-1}$ ) is evident, in particular, in the region below $m_{\phi}$ the sensitivity is much better than what can be achieved at Belle-II.

- LHCb has already produced results [13] that shows the ability to identify c-jets vs b-jets. For instance, for a jet with $\mathrm{P}_{\mathrm{T}}>20 \mathrm{GeV}$ a c-jet tag efficiency of 6(20)\% is achieved for a b-jet tag efficiency of $1(10) \%$. If this level of discrimination is maintained in the second upgrade, the increase of luminosity may compensate for the low LHCb acceptance for Higgs decays, and there is a very interesting potential to search for Higgs decaying into charm quarks. The precision achieved on the Higgs Yukawa couplings to charm should be very close to that required to reach the small values predicted by the SM. 


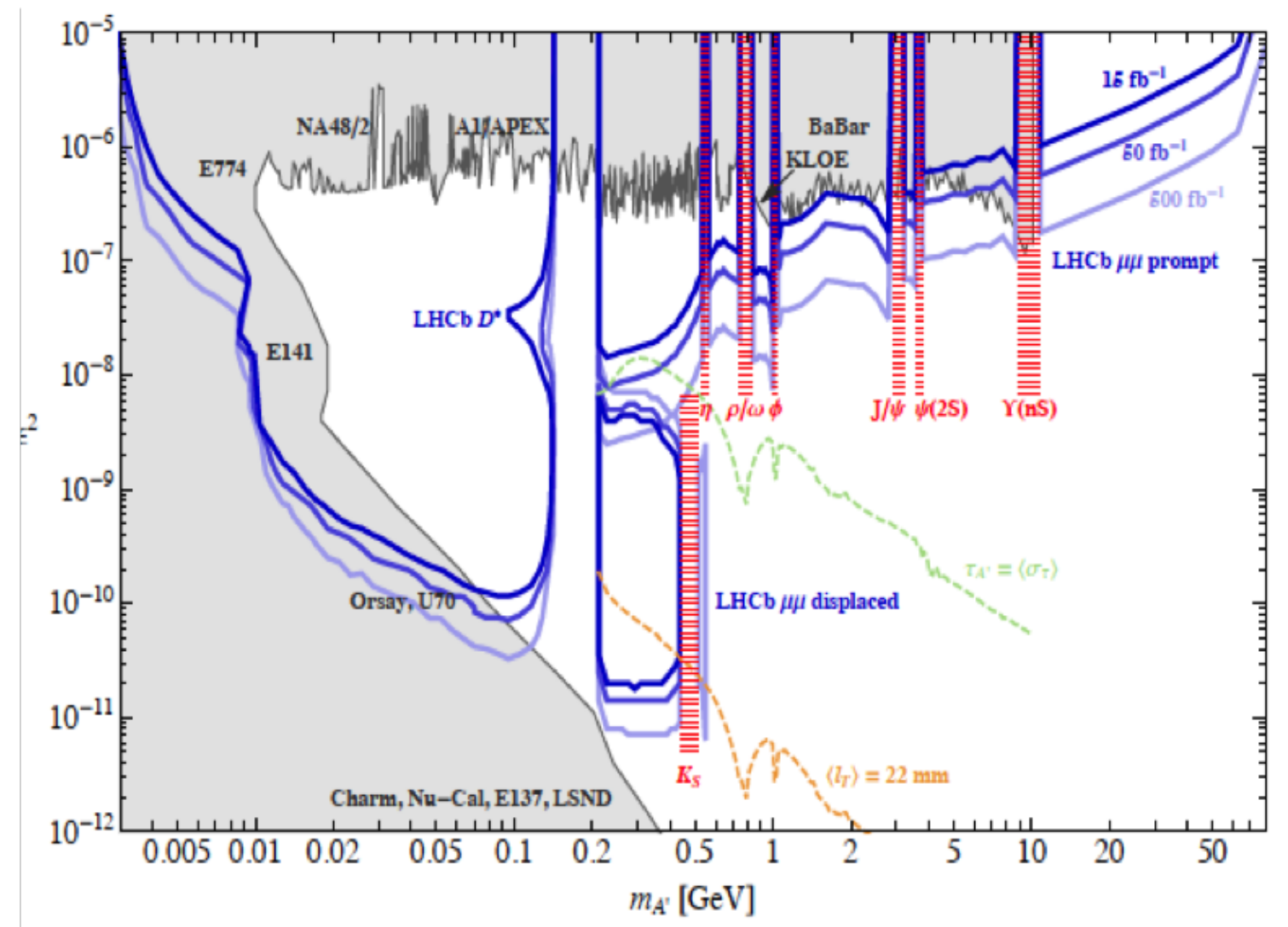

Figure 1: Bounds on dark photons (A') mass (x-axis) and coupling (y-axis) compared to the anticipated $\mathrm{LHCb}$ reach for inclusive $\mathrm{A}^{\prime}$ production in the di-muon channel. The contours assume an integrated luminosity of: $16,50,500 \mathrm{fb}^{-1}$. This figure is taken from reference [12] where a more detailed description of the different contributions to this figure can be found.

\section{HL-LHC and IR8}

While it is hoped that the previous section has convinced the reader that it is indeed a good physics opportunity to consider a second upgrade for $\mathrm{LHCb}$, the first questions we need to answer are if HL-LHC can deliver enough luminosity for a third high luminosity experiment, and if the Interaction Region where LHCb sits (IR8) can be adapted for such an experiment.

During RUN4 HL-LHC will be delivering a maximum instantaneous luminosity to ATLAS/CMS (LHCb) of $\sim 20(1.1) \times 10^{34} \mathrm{~Hz} \mathrm{~cm}^{-2}$ which will be levelled to an average luminosity of $\langle\mathscr{L}\rangle$ $\sim 5(0.2) \times 10^{34} \mathrm{~Hz} \mathrm{~cm}^{-2}$. If LHCb simply does not level the luminosity, and all the rest of the HL-LHC parameters are left untouched, then it is expected to increase the annual integrated luminosity from $10 \mathrm{fb}^{-1} /$ year in RUN4 to $30 \mathrm{fb}^{-1} /$ year in RUN5 at LHCb. To go beyond this, the optics in IR8 need to be changed, namely a reduction of the nominal $\beta^{*}=3 \mathrm{~m}$. A scenario with an initial instantaneous luminosity of $\sim 2 \times 10^{34} \mathrm{~Hz} \mathrm{~cm}^{-2}$ in IR8 with $\beta^{*}=2 \mathrm{~m}$ has been studied [14] and could provide $40 \mathrm{fb}^{-1} /$ year, while going down to $\beta^{*}=1 \mathrm{~m}$ may well be feasible and could potentially provide up to $70 \mathrm{fb}^{-1} /$ year. In all these scenarios studied the effect of proton burn-off reducing the luminosity delivered to ATLAS/CMS is found to be negligible at the few percent level. 
IR8 is not designed to host a high luminosity experiment. In particular, one of the most challenging and costly project of HL-LHC is the design of the much larger aperture $(150 \mathrm{~mm})$ inner triplet magnets for the interaction regions of ATLAS and CMS, as the present ones cannot accumulate more than $\sim 300 \mathrm{fb}^{-1}$ and are a limitation to the maximum instantaneous luminosity. Preliminary studies have shown that up to these integrated luminosities, without having to replace the LHCb inner triplet magnets, they can be effectively protected by additional shielding (Target Absorber of Neutrals, TAN and Long Collimators, TCL) as is the case at ATLAS and CMS. Moreover, the first compensator magnet could be filled with Tungsten and act as Target Absorber of Secondaries (TAS). Although more work is needed in this area, so far no showstoppers have been identified [14].

\section{The detector challenge}

Even though it seems possible for HL-LHC to increase the integrated luminosity delivered to $\mathrm{LHCb}$ to reach $300 \mathrm{fb}^{-1}$, the $\mathrm{LHCb}$ detector will clearly need to be modified to deal with an instantaneous luminosity of up to $2 \times 10^{34} \mathrm{~Hz} \mathrm{~cm}^{-2}$ and a pileup of up to $\mu \sim 50$. Preliminary studies have indicated already some possible solutions.

The current design of the Vertex Detector [15] (VELO) for the first upgrade could survive this level of occupancies by increasing the inner radius from 5 to $11 \mathrm{~mm}$, at the cost of a degradation in the impact parameter resolution, from $50 \%$ to $100 \%$ depending on the track pseudorapidity. A better solution, from the point of view of physics performance, would be to replace the VELO sensors with thinner detectors, smaller pixels and new electronics. In average, $\sim 13 \%$ of B decays will be mismatched to the wrong PV with an average pileup of $\mu \sim 50$, which will limit the possibilities to perform time-dependent $\mathrm{CP}$ asymmetries, as well as increase the background levels. If the time information of the track hit is known within a resolution of $\sim 200 \mathrm{ps}$, the mismatch is reduced to the current levels (1\%). See Figure 2 for a preliminary study on how this fraction depends on the hit time resolution. This study clearly motivates the use of timing information in the VELO, although the radiation resistance is an open issue to be further studied.

The SciFi tracker [16] being built now for the first upgrade is limited to work not beyond instantaneous luminosities of $2 \times 10^{33} \mathrm{~Hz} \mathrm{~cm}^{-2}$ given the radiation and occuppancies expected. A possible solution (although not cheap) is to replace the tracker by a full Silicon Tracker for example using technologies developed for the ATLAS and CMS upgrades.

As mentioned in Section 1 the current plan is to replace the inner region of ECAL [17] during LS3 due to radiation damage. It turns out that by just improving the resolution in the horizontal band ( $\pm 80 \mathrm{~cm}$ in the $\mathrm{y}$-axis) the resolution achieved in radiative $\mathrm{B}$ decays is very close to the improvement replacing the whole area of the calorimeter $( \pm 300 \mathrm{~cm}$ in the $\mathrm{y}$-axis). Therefore, it seems like a good option to use some of the new technologies being studied (W-Shaslik, DECAL, etc...) on just this horizontal band to provide a better detector able to run at higher luminosities. Preliminary studies indicate a factor 2 better resolution in position and a factor 4 on pileup reduction.

The LHCb muon system [17] will suffer from a too high rate in the innermost regions of some of the Muon stations (mainly M2 and M3). Replacing HCAL by an iron wall to act as shielding would reduce rates by a factor 2 , but still new technologies (like $\mu$-RWELL, GEM, etc...) will be 


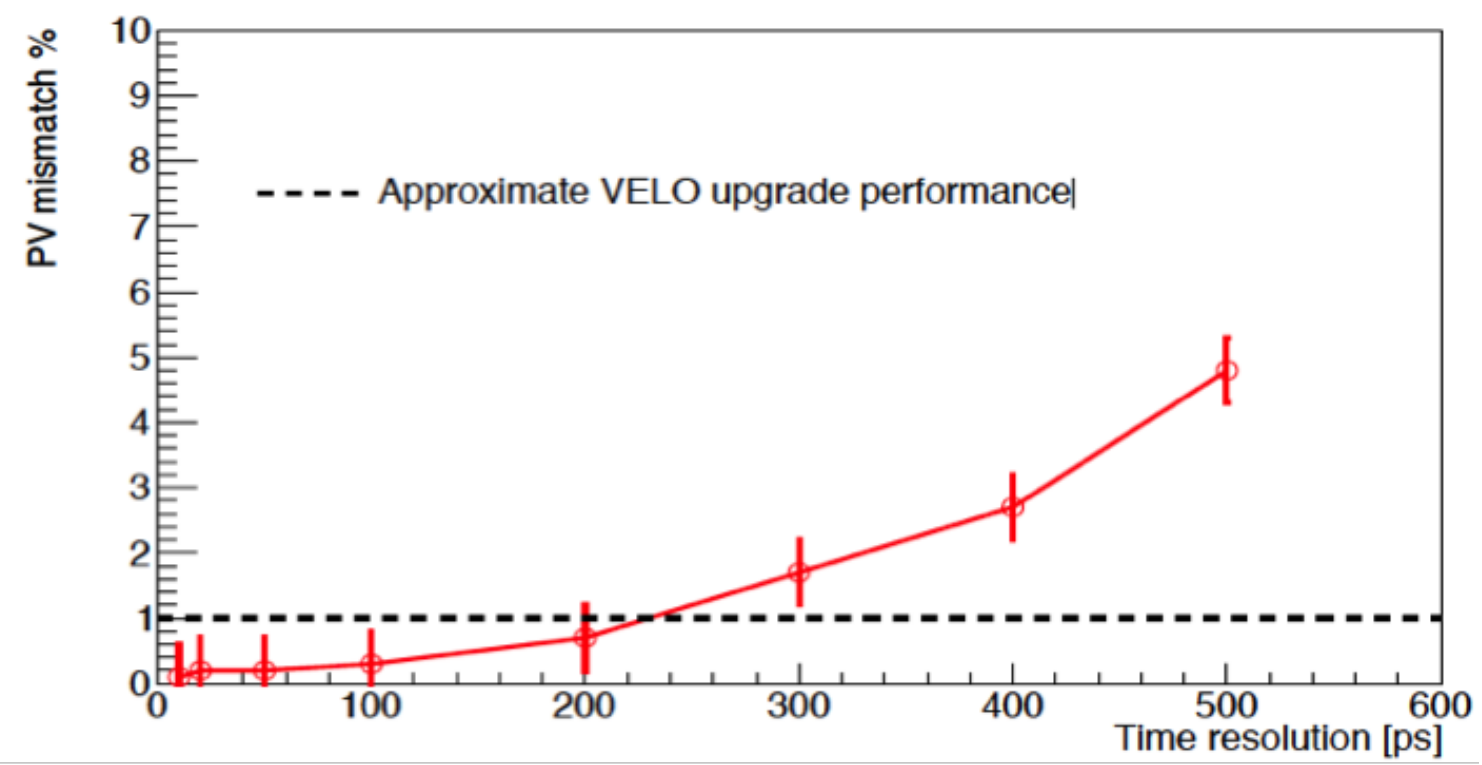

Figure 2: Fraction of B decays mismatch to the wrong PV as a function of the resolution in time of the VELO hits obtained from a sample of simulated events with instantaneous luminosities of $2 \times 10^{34} \mathrm{~Hz} \mathrm{~cm}^{-2}$.

needed to replace the innermost detectors. Moreover, the muon-id algorithm can probably relax the requirements in terms of hit coincidences in different stations and still have a very high purity.

It was initially thought that the RICH system [17] would find the particle densities an insurmountable challenge. However, after a preliminary study it does seem to be possible to reduce by a factor 10 occupancies simply by increasing the pixel granularity ( $3 \mathrm{~mm}$ down to $1 \mathrm{~mm}$ ) and using photodetectors insensitive to magnetic field (i.e. SiPM or MCP) therefore removing the need for shielding. If in addition, the optical uncertainty is reduced by moving the flat light-weight mirror into the acceptance and by reducing the mirror tilt (hence reducing the emission point uncertainty) it will be possible to correctly identify the Cherenkov rings. Moreover the chromatic uncertainty can be reduced by the tuning of the gas and the use of photodetectors shifted towards the red spectrum.

Although no full design yet exist, it does seem from a preliminary look at the main detector challenges that solutions can be found.

\section{Final comments}

It seems clear that HL-LHC can provide up to $2 \times 10^{34} \mathrm{~Hz} \mathrm{~cm}^{-2}$ instantaneous luminosity to IR8 without significant loses for the other interaction points at the LHC ring, and be able to integrate up to $300 \mathrm{fb}^{-1}$ before the insertion magnets at IR8 need replacement. The need for extra protecting devices seems to be limited and affordable until then.

There is a clear and strong physics case for an LHCb second upgrade in LS4. Very rare decays, or exotic searches are limited by statistics. Some of the most interesting CKM measurements are not limited by systematic uncertainties. In particular, time dependent CP asymmetry measurements seem feasible if tracker detectors have timing information $(\sim 200 \mathrm{ps}$ resolution per hit). 
Some of the improved detector technologies needed for this second upgrade, and other ideas being discussed, could already be implemented during LS3 (phase 1b upgrade).

No doubt the $\mathrm{LHCb}$ phase 2 upgrade is a challenging project, but so far LHCb has been very successful in beating expectations!

\section{References}

[1] G. Ciezarek, H. M. Evans, B. Khanji, M. J. Morello, K. Petridis, Z. Xu, Contributions to these proceedings.

[2] F. Cheung, L. Eklund, S.C. Haines, J.A. De Vries, C. Vazquez, Contributions to these proceedings.

[3] D. C. Craik, Contribution to these proceedings.

[4] G. Simi, Contribution to these proceedings.

[5] CERN-Council-S/106, http://council.web.cern.ch/council/en/EuropeanStrategy/esc-e-106.pdf

[6] M.J. Charles and R. Forty, TORCH: Time of Flight Identification with Cherenkov Radiation, Nucl. Instrum. Meth. A 639 (2011) 173 [arXiv: 1009.3793]

[7] V. Khachatryan, et al. (CMS and LHCb Collaborations), Observation of the rare $B_{s}^{0} \rightarrow \mu^{+} \mu^{-}$decay from the combined analysis of CMS and LHCb data, Nature 522 (2015) 68-72 [arXiv:1411.4413]

[8] CMS Collaboration, Study of $B \rightarrow \mu^{+} \mu^{-}$decays as a physics benchmark for the CMS Phase-II upgrade, CMS PAS FTR-14-015.

[9] R. Aaij, et al. (LHCb Collaboration), Search for the lepton flavour violating decay $\tau^{-} \rightarrow \mu^{-} \mu^{+} \mu^{-}$, 10.1007/JHEP02(2015)121 [arXiv: 1409.8548]

[10] J.P. Lees et al. (BaBar Collaboration), Limits on $\tau$ lepton-flavour violating decays intro three charged leptons, Phys. Rev. D 81, (2010) 111101(R) [arXiv:1002.4550].

K.Hayasaka et al. (Belle Collaboration), Search for lepton-flavour-violating $\tau$ decays into three leptons with 719 million produced $\tau^{+} \tau^{-}$pairs, Phys. Lett. B 687, (2010), 139.

[11] P. Paradisi, JHEP 0510, 006 (2005).

K.S.Babu and C. Kolda, Phys. Rev. Lett. 89, 241802 (2002).

A. Brignole and A. Rossi, Phys. Lett. B 566, 217 (2003).

[12] P. Ilten, Y. Soreq, J. Thaler, M. Williams and W. Xue, Proposed Inclusive Dark Photon Search at LHCb Phys. Rev. Lett. 116, 251803 (2016) [arXiv:1603.08926]

[13] R. Aaij, et al. (LHCb Collaboration), Identification of beauty and charm quark jets at LHCb JINST 10 (2015) P06013 [arXiv:1504.07670]

[14] $5^{\text {th }}$ Joint HiLumi LHC-LARP Annual Meeting, October (26-30) (2015), CERN, Geneva, [https://espace.cern.ch/Hilumi/2015].

[15] R. Aaij, et al. (LHCb Collaboration), LHCb VELO Upgrade Technical Design Report CERN-LHCC-2013-021; LHCb-TDR-013.

[16] R. Aaij, et al. (LHCb Collaboration), LHCb Tracker Upgrade Technical Design Report CERN-LHCC-2014-001; LHCb-TDR-015.

[17] R. Aaij, et al. (LHCb Collaboration), LHCb PID Upgrade Technical Design Report CERN-LHCC-2013-022; LHCb-TDR-014. 